\title{
Erratum to: Distribution of metals and quality of intertidal surface sediment near commercial ports and estuaries of urbanized rivers in Port Klang, Malaysia
}

\author{
Hazzeman Haris • Ahmad Zaharin Aris
}

Published online: 25 December 2014

(c) Springer-Verlag Berlin Heidelberg 2014

\section{Erratum to: Environ Earth Sci}

\section{DOI 10.1007/s12665-014-3900-7}

The original article has been published inadvertently with some errors in Table 4 . The corrected Table 4 is given below.

Table 4 Correlation coefficient between metals, LOI and sediment particle size (clay, silt and sand) based on Kendall's tau-b correlation analysis

\begin{tabular}{|c|c|c|c|c|c|c|c|c|c|c|c|c|}
\hline & LOI & $\mathrm{Cd}$ & Co & $\mathrm{Cu}$ & $\mathrm{Cr}$ & $\mathrm{Mn}$ & $\mathrm{Ni}$ & $\mathrm{Pb}$ & $\mathrm{Zn}$ & Clay & Silt & Sand \\
\hline LOI & 1.000 & & & & & & & & & & & \\
\hline $\mathrm{Cd}$ & $0.610 * *$ & 1.000 & & & & & & & & & & \\
\hline Co & $0.446 * *$ & $0.454 * *$ & 1.000 & & & & & & & & & \\
\hline $\mathrm{Cu}$ & $0.577 * *$ & $0.760 * *$ & $0.421 * *$ & 1.000 & & & & & & & & \\
\hline $\mathrm{Cr}$ & $0.447 * *$ & $0.580 * *$ & $0.651 * *$ & $0.576^{* *}$ & 1.000 & & & & & & & \\
\hline $\mathrm{Mn}$ & $0.298 * *$ & $0.305^{* *}$ & $0.582 * *$ & $0.293 * *$ & $0.389 * *$ & 1.000 & & & & & & \\
\hline $\mathrm{Ni}$ & $0.512 * *$ & $0.587 * *$ & $0.673 * *$ & $0.588^{* *}$ & $0.695 * *$ & $0.459 * *$ & 1.000 & & & & & \\
\hline $\mathrm{Pb}$ & $0.575 * *$ & $0.735 * *$ & $0.516^{* *}$ & $0.819 * *$ & $0.621 * *$ & $0.358 * *$ & $0.680 * *$ & 1.000 & & & & \\
\hline $\mathrm{Zn}$ & $0.523^{* *}$ & $0.721 * *$ & $0.444 * *$ & $0.751 * *$ & $0.508 * *$ & $0.295 * *$ & $0.583 * *$ & $0.756^{* *}$ & 1.000 & & & \\
\hline Clay & $0.478 * *$ & $0.338 * *$ & $0.430 * *$ & $0.310^{*}$ & $0.315^{*}$ & $0.389 * *$ & $0.393 * *$ & $0.278^{*}$ & $0.402 * *$ & 1.000 & & \\
\hline Silt & $0.499 * *$ & $0.329 *$ & $0.503 * *$ & $0.283^{*}$ & $0.315^{*}$ & $0.444 * *$ & $0.421 * *$ & $0.324 *$ & $0.283^{*}$ & $0.669 * *$ & 1.000 & \\
\hline Sand & $-0.504 * *$ & $-0.329 *$ & $-0.439 * *$ & $-0.283 *$ & $-0.287 *$ & $-0.389 * *$ & $-0.375^{* *}$ & $-0.287 *$ & $-0.338 * *$ & $-0.845^{* *}$ & $-0.824 * *$ & 1.000 \\
\hline
\end{tabular}

* Correlation is significant at the 0.05 level (2-tailed)

** Correlation is significant at the 0.01 level (2-tailed)

The online version of the original article can be found under doi:10.1007/s12665-014-3900-7.

H. Haris · A. Z. Aris $(\square)$

Faculty of Environmental Studies, Environmental Forensics

Research Centre, Universiti Putra Malaysia, UPM,

43400 Serdang, Selangor, Malaysia

e-mail: zaharin@env.upm.edu.my

H. Haris

School of Biological Sciences, Universiti Sains Malaysia, USM,

11800 Penang, Malaysia 